\title{
Antibioticoterapia em crianças indígenas e não indígenas desnutridas
}

\section{Antibiotic therapy in indigenous and non-indigenous undernourished children}

Naipy Abreu Brunozi ${ }^{1}$ Flávia Lúcia David²

'Universidade Federal de Mato Grosso (UFMT) - Rondonópolis (MT), Brasil.

2UFMT - Araguaia (MT), Brasil.

\section{RESUMO}

Introdução: A prevalência da desnutrição infantil vem diminuindo em todo o mundo, mas ainda acomete milhões de crianças, especialmente indígenas. Devido ao elevado número de doenças infecciosas associadas à desnutrição, a antibioticoterapia faz parte da terapêutica recomendada. Objetivo: Observar os casos de desnutrição entre crianças indígenas e não indígenas hospitalizadas e a terapêutica empregada durante o tratamento. Métodos: Estudo de coorte retrospectivo, farmacoepidemiológico, realizado com informações extraídas de prontuários arquivados do período de janeiro de 2012 a dezembro de 2014 de um hospital público. Resultados: Participaram 166 crianças, sendo o número de crianças indígenas aproximadamente seis vezes maior do que não indígenas. Houve maior prevalência entre lactentes e crianças com idade inferior a um ano apresentaram mais chances de serem internadas por desnutrição. Os diagnósticos de desnutrição mais vistos foram os inespecíficos, com uma proporção significativa de óbitos relacionados ao diagnóstico E43. As infecções mais comuns foram do sistema digestório e respiratório. Crianças indígenas tiveram quase cinco vezes mais chances de apresentarem infecção respiratória. A maior proporção recebeu até três antibióticos, havendo crianças que receberam mais que sete antibióticos diferentes durante o período de internação. Conclusão: A população infantil deve ser acompanhada por meio de inquéritos que possam subsidiar políticas de saúde que atendam suas necessidades. É necessária a capacitação dos profissionais envolvidos no cuidado da criança desnutrida, recursos materiais e financeiros, a fim diminuir o número de diagnósticos inespecíficos e evitar o uso indiscriminado de antibióticos, sendo imprescindível uma política de controle efetiva no uso da politerapia antimicrobiana.

Palavras-chave: desnutrição; desenvolvimento infantil; índios SulAmericanos; Saúde de populações indígenas; antibacterianos; resistência microbiana a medicamentos.

\begin{abstract}
Introduction: The prevalence of child malnutrition is declining worldwide, but still affects millions of children, especially indigenous people. Due to the high number of infectious diseases associated with malnutrition, antibiotic therapy is part of the recommended therapy. Objective: To observe the cases of malnutrition among hospitalized indigenous and non-indigenous children and the therapy used during treatment. Methods: Retrospective cohort study, pharmacoepidemiological, carried out with information extracted from medical records filed from January 2012 to December 2014 of a public hospital. Results: 166 children participated, with the number of indigenous children being approximately six times higher than that of nonindigenous children. There was a higher prevalence among infants and children under one year of age who were more likely to be hospitalized for malnutrition. The most frequent diagnoses of malnutrition were nonspecific, with a significant proportion of deaths related to diagnosis E43. The most common infections were of the digestive and respiratory system. Indigenous children were almost five times more likely to have respiratory infection. The highest proportion received up to three antibiotics, with children receiving more than seven different antibiotics during the hospitalization period. Conclusion: The child population must be accompanied by surveys that can subsidize health policies that meet their needs. It is necessary to train the professionals involved in the care of malnourished children, material and financial resources, in order to reduce the number of non-specific diagnoses and to avoid the indiscriminate use of antibiotics, a policy of effective control in the use of antimicrobial polytherapy is essential.
\end{abstract}

Keywords: malnutrition; child development; indians, South American; health of indigenous peoples; anti-bacterial agents; drug resistance, microbial.

Recebido em: 12/03/2018

Revisado em: $23 / 08 / 2018$

Aprovado em: 21/11/2018

Autor para correspondência: Naipy Abreu Brunozi - Universidade Federal de Mato Grosso - Avenida dos Estudantes, 5055, Bairro Sagrada Família CEP: 78735-910 - Rondonópolis - (MT), Brasil - E-mail: nabrunozi@hotmail.com

Conflito de interesses: nada a declarar 


\section{INTRODUÇÃO}

O número de crianças desnutridas em todo o mundo caiu quase pela metade ao longo das duas últimas décadas, entretanto, estima-se ainda que uma em cada sete permaneça abaixo do peso, somando aproximadamente mais de 90 milhões de crianças ${ }^{1}$.

No Brasil, o número de casos de desnutrição também apresentou reduções significativas, mas ainda permanecem elevadas proporções, especialmente entre a população indígena, o que evidencia a discrepância entre as condições de vida e saúde entre o Brasil indígena e o não indígena ${ }^{2}$.

A desnutrição é uma causa importante de morbidade e mortalidade e está frequentemente associada a doenças infecciosas que complicam o quadro clínico e favorecem o óbito. Devido à elevada prevalência de infecções associadas, a Organização Mundial de Saúde (OMS) recomenda a prescrição de rotina de antibióticos para crianças com desnutrição aguda grave, diante da possibilidade desta intervenção reduzir significativamente a mortalidade ${ }^{3}$. Contudo, essa prática é questionada devido à lacuna de ensaios clínicos que demonstrem sua eficácia, especialmente na ausência de complicações associadas e diante de ameaça da resistência antimicrobiana ${ }^{4}$.

Sustenta-se ainda essa conduta terapêutica, pois é frequente no caso de crianças severamente desnutridas, que não apresentem sinais e sintomas clássicos de infecção devido ao comprometimento do sistema imunológico. No entanto, a dúvida quanto à prescrição antimicrobiana rotineira permanece devido à ausência de evidências de qualidade na literatura que sustentem tal conduta terapêutica, há poucos ensaios clínicos que apontam para uma redução da mortalidade em crianças que receberam antibioticoterapia. Existe, portanto, a necessidade urgente de boas evidências clínicas que sustentem as recomendações atuais ${ }^{3,5}$.

Logo, o objetivo desse estudo foi observar os casos de desnutrição entre crianças indígenas e não indígenas hospitalizadas e a terapêutica empregada durante o tratamento.

\section{MÉTODOS}

Trata-se de um estudo de coorte retrospectivo, farmacoepidemiológico, não aleatorizado. Foi realizado a partir de prontuários arquivados, oriundos do setor de pediatria de um hospital público de Mato Grosso, Brasil.

A pesquisa foi realizada em um hospital integrado ao Sistema Único de Saúde (SUS) que realiza atendimentos de média e alta complexidade, sendo referência para os municípios de uma das microrregiões de saúde do estado, no entanto, devido à sua localização geográfica, em região de divisa estadual e a presença de rodovias importantes no município onde se encontra, o hospital recebe usuários de todas as localidades do país. É ainda, referência para o Distrito Sanitário Especial Indígena (DSEI) Xavante, atendendo tanto a população indígena como não indígena.
A população do estudo foi composta a partir de informações de prontuários de crianças indígenas e não indígenas com desnutrição, internadas no período de janeiro de 2012 a dezembro de 2014, sendo a faixa etária designada de zero até cinco anos.

As internações por desnutrição foram selecionadas por meio do espelho da Autorização de Internação Hospitalar (AIH) anexa aos prontuários considerando o item "Diagnóstico principal", apresentado conforme a Classificação Internacional de Doenças (CID-10), sendo incluídas todas as crianças com os seguintes diagnósticos: (E 40) Kwashiorkor; (E 41) Marasmo nutricional; (E 42) Kwashiorkor; (E 43) Desnutrição proteico-calórica grave não especificada; (E 44) Desnutrição proteico-calórica de graus moderado e leve; (E 44.0) Desnutrição proteico-calórica moderada; (E 44.1) Desnutrição proteico-calórica leve; (E 45) Atraso do desenvolvimento devido a desnutrição proteico-calórica; e (E 46) Desnutrição proteico-calórica não especificada.

Também foram incluídos os prontuários de pacientes em que, apesar de não apresentarem desnutrição como diagnóstico principal, a condição foi apontada como diagnóstico secundário nas evoluções médicas. Foram excluídos apenas os prontuários cujo espelho da AIH não estava anexado.

As informações foram coletadas na sala de pesquisa do Serviço de Arquivo Médico e Estatística (SAME) da instituição, onde foram feitos registros fotográficos (Formato JPEG) dos prontuários que atenderam aos critérios de inclusão.

Após a coleta de dados, todos os prontuários foram lidos e as seguintes informações foram extraídas: sexo, raça/cor, etnia, diagnóstico principal, data de internação e saída, desfecho clínico, registros de doenças infecciosas e antibióticos prescritos. Foi criado um banco de dados no software Epi Info versão 3.5.2 e realizada a digitação dos dados, que posteriormente foram exportados e analisados no software Statistical Package for the Social Sciences (SPSS) for Windows versão 20.

Para a análise estatística, quando necessário, foram realizados testes não-paramétricos (Chi-quadrado de Pearson e Exato de Fischer) e nos casos aplicáveis Razão de Chances (Odds Ratio), com intervalo de confiabilidade de $95 \%$, sendo considerado estatisticamente significante o valor $p$-associado menor ou igual a $0,05(p \leq 0,05)$.

Esse estudo faz parte do projeto de pesquisa "Farmacoepidemiologia da Prescrição e Dispensação de Medicamentos na Região do Médio Araguaia - MT", aprovado pelo Comitê de Ética em Pesquisa do Hospital Universitário Júlio Müller com número do processo: 987/CEP-HUJM/2011.

\section{RESULTADOS E DISCUSSÃO}

Ao longo dos três anos analisados foram identificados 166 prontuários de crianças internadas com desnutrição, sendo 141 $(84,9 \%)$ indígenas e $25(15,1 \%)$ não indígenas. O número de 
crianças indígenas desnutridas foi aproximadamente seis vezes maior do que o não indígena. A etnia Xavante foi a única encontrada, o que certamente ocorreu, pois, o hospital onde os dados foram coletados também é referência para o DSEI Xavante.

A etnia Xavante se destaca, em meio às insatisfatórias condições da saúde indígena no Brasil, com elevadas taxas de mortalidade infantil, casos de doenças infecciosas e frequência da desnutrição, contribuindo como evidência de disparidades entre o Brasil indígena e não indígena ${ }^{6}$. A desnutrição crônica, caracterizada pelo déficit estatural que corresponde ao índice altura-para-idade, se sobressai entre a etnia e, de maneira geral, em outras comunidades indígenas, como apontado no I Inquérito Nacional de Saúde e Nutrição Indígena e em outros estudos, superando médias nacionais ${ }^{7,8}$.

Durante a realização desse estudo, não foi possível trabalhar os índices altura-para-idade, peso-para-altura e peso-para-idade, devido à ausência de registro da estatura e, às vezes, do peso dos pacientes nos prontuários. Entretanto, a maior prevalência de desnutrição em crianças indígenas durante o período analisado, corrobora com as informações encontradas na literatura.

Os hábitos alimentares são claramente influenciados por questões socioeconômicas e culturais, e no caso dos pacientes indígenas, há de se considerar que as situações econômica, habitacional e de saneamento básico inadequadas, destacadas pelo Censo Demográfico de 2010, contribuem para a maior prevalência da desnutrição entre suas crianças".

No caso da etnia Xavante, sua alimentação está ligada a processos culturais, simbólicos e cosmológicos na vinculação dos Xavantes à natureza, numa noção de pertencimento ao território. No entanto, com a ocupação de terras no estado do Mato Grosso pela pecuária e a agricultura, as terras Xavante se tornaram ilhas de biodiversidade, restringindo práticas que lhes propiciavam meios para subsistência. Esta situação corrobora com a insegurança alimentar, desnutrição e elevada taxa de mortalidade infantil entre esta população, relacionada a um problema basilar que é o acesso à terra de modo que permita a vivência dos indígenas conforme sua cosmologia, ou seja, uma estreita ligação entre o Xavante e a natureza ${ }^{10}$. A dimensão das terras indígenas é um elemento central para sobrevivência física e cultural de diversas etnias, logo, intrinsecamente ligada à alimentação e estado nutricional ${ }^{9}$.

No que diz respeito ao sexo, esse não apareceu como um determinante do estado nutricional $(\mathrm{p}>0,05)$. A distribuição de meninos e meninas entre os grupos indígena e não indígena foi próxima, não havendo diferença superior a dois por cento.

Analisando-se as faixas etárias, foi visto que a maior proporção de crianças desnutridas, $78,3 \%$ da amostra, esteve entre os lactentes ( $\geq 28$ dias e $<$ dois anos). Outros estudos também mostram maior proporção de desnutrição e risco de desnutrição entre pacientes com idade inferior a dois anos, inclusive entre indígenas, como já foi descrito maiores déficit dos índices altura-para-idade e peso-para-idade em lactentes indígenas Guarani, especificamente na faixa etária de 12 a 23 meses $^{7,11}$.

A alimentação adequada nos primeiros dois anos de vida é essencial para o desenvolvimento saudável da criança, sendo que os momentos críticos são os primeiros seis meses, quando o aleitamento materno deve ser exclusivo, e ao findar os seis meses, deve ser introduzida a alimentação complementar, tendo o cuidado para que atenda todas as necessidades nutricionais da criança. Nos países em desenvolvimento o desmame precoce comum, o que contribui para a morbimortalidade infantil, e referente a introdução da alimentação complementar, quando esta acontece precocemente, contribui para infecções e desnutrição ${ }^{12}$. Logo, a ausência do aleitamento materno e a introdução de uma alimentação complementar que não responda adequadamente as demandas energéticas da criança podem justificar a maior proporção da desnutrição entre os lactentes.

Visto que a maior parte dos pacientes era lactente, foi feita a análise da seguinte classificação etária: crianças até doze meses e com mais de doze meses de vida (Tabela 1). A partir dessa classificação, foi possível ver que as crianças com idade inferior a doze meses têm 2,64 vezes mais chances de serem internadas com desnutrição do que aquelas com idade superior $(\mathrm{p}<0,05)$. A ausência de um conceito claro sobre amamentação exclusiva e uma perspectiva incorreta sobre alimentação complementar podem contribuir para essa realidade, nesse contexto a atuação de profissionais de saúde durante o pré-natal e puerpério pode ter um impacto significativo contribuindo para a redução desses números e outras causas de morbidade infantil ${ }^{13}$.

Sobre as causas de hospitalização, estudos apontam que as doenças do aparelho respiratório são as mais frequentes entre crianças menores de cinco anos, tanto entre indígenas como não indígenas, sendo frequentemente seguidas por doenças infecciosas e parasitárias ${ }^{14,15}$. Visto que este estudo objetivou a análise de prontuários apenas de crianças que apresentaram o diagnóstico de desnutrição, ainda que em alguns casos tenha sido o diagnóstico secundário, as causas de hospitalização mais frequentes foram as doenças endócrinas nutricionais e metabólicas, seguidas por doenças do aparelho respiratório e doenças infecciosas e parasitárias (Tabela 2).

Tabela 1: Classificação dos pacientes pediátricos com desnutrição, não indígenas e indígenas, segundo faixa etária $(n=166)$. Mato Grosso, Brasil, 2012-2014.

\begin{tabular}{|c|c|c|c|c|c|c|c|}
\hline \multirow{3}{*}{ Indígena } & \multicolumn{6}{|c|}{ Faixa Etária } & \multirow{3}{*}{ Estatística } \\
\hline & \multicolumn{2}{|c|}{$\leq 12$ meses } & \multicolumn{2}{|c|}{$>12$ meses } & \multicolumn{2}{|c|}{ Total } & \\
\hline & (f) & $\%$ & (f) & $\%$ & (f) & $\%$ & \\
\hline Não & 15 & 9 & 10 & 6 & 25 & 15,1 & \multirow{3}{*}{$\begin{array}{l}\text { OR } 2,64 \\
\chi^{2}=5,28 \\
p=0,022\end{array}$} \\
\hline Sim & 51 & 30,8 & 90 & 54,2 & 141 & 84,9 & \\
\hline Total & 66 & 39,8 & 100 & 60,2 & 166 & 100 & \\
\hline
\end{tabular}

$\mathrm{n}=$ número; $(\mathrm{f})=$ quantidade; \%=porcentagem; OR: Odds Ratio; $\chi^{2}=$ Chi-quadrado de Pearson. 
No que tange à saúde indígena, Lunardi et al. ${ }^{16}$, ao analisarem prontuários de indígenas Xavante internados no período de 2000 a 2002, verificaram que entre os menores de cinco anos as causas de hospitalização mais frequentes foram: doenças do aparelho respiratório, algumas doenças infecciosas e parasitárias e doenças endócrinas, nutricionais e metabólicas, nesta ordem. A diferença desses dados para com os encontrados neste estudo pode ser atribuída ao maior enfoque dado à desnutrição. Observa-se, então, que as causas de morbidade e internação entre as crianças Xavante menores de cinco anos permanecem as mesmas ao longo dos anos.

Trabalhando-se apenas os diagnósticos de desnutrição, foram encontrados 100 pacientes que apresentaram alguma forma de desnutrição como motivo principal de internação (Tabela 3). Chamase a atenção para o fato de que os diagnósticos mais aplicados foram os inespecíficos, E43: Desnutrição proteico-calórica grave não especificada, e E46: Desnutrição proteico-calórica não especificada.

No Brasil, o Ministério da Saúde publicou o "Manual de Atendimento da Criança com Desnutrição Grave em Nível Hospitalar", e na época das discussões para a sua elaboração, uma das necessidades que ficou evidente foi a de aprimorar o diagnóstico da população infantil internada, isso porque foi visto que o diagnóstico de desnutrição mais frequente foram os inespecíficos, que havia uma subestimação da desnutrição grave, e elevada letalidade associada ao diagnóstico E43 em crianças de doze meses a cinco anos ${ }^{17}$.

A importância do aprimoramento do diagnóstico, com o propósito da diminuição da morbimortalidade infantil, também pode ser vista neste estudo, pois ao ser verificada a proporção de óbitos entre os diagnósticos, foi notado que sua distribuição não foi homogênea, havendo maior proporção de óbitos relacionados ao diagnóstico E43: Desnutrição proteico-calórica grave não especificada $(\mathrm{p}<0,05)$ (Tabela 4). Resultado semelhante também foi encontrado no México ao ser analisada a mortalidade por desnutrição em menores de cinco anos no período de 1990 a 2009. Conforme a pesquisa, nesse período houve maior número de mortes nos casos de desnutrição de caráter impreciso, cerca de $54 \%$ dos óbitos registrados ${ }^{18}$.

De maneira geral, 155 (93,8\%) dos pacientes evoluíram para alta. Houve sete óbitos, dos quais seis foram indígenas. Os demais pacientes foram transferidos. Apesar do pequeno número de óbitos registrados, a desnutrição não deve ser ignorada, pois pode comprometer o desenvolvimento físico e cognitivo irreversivelmente, além de estar associada a doenças infecciosas que contribuem com o agravo nutricional e aumento da mortalidade infantil ${ }^{19}$.

A relação entre a desnutrição e doenças infecciosas é bem documentada na literatura, o que torna sua avaliação importante, considerando-se ainda que a criança desnutrida pode não apresentar sinais comuns de infecção, como a hipertermia ${ }^{3,19}$.

Dentre os pacientes desse estudo, $147(88,6 \%)$ tiveram pelo menos uma infecção, dos quais 127 eram indígenas. As infecções mais frequentes foram àquelas relacionadas ao sistema digestório, como pode ser visto na Tabela 5, entretanto, não houve diferença estatística significante entre os grupos indígena e não indígena $(p>0,05)$. A ausência de diferença estatística nos casos apresentados pode ser atribuída à disparidade entre o número de crianças

Tabela 2: Classificação dos pacientes pediátricos, indígenas e não indígenas, conforme o diagnóstico principal ( $n=166)$. Mato Grosso, Brasil, 2012-2014

\begin{tabular}{|c|c|c|c|c|c|c|}
\hline \multirow[t]{2}{*}{ Diagnóstico principal segundo a CID - 10} & \multicolumn{2}{|c|}{$\begin{array}{c}\text { Não } \\
\text { indígena }\end{array}$} & \multicolumn{2}{|c|}{ Indígena } & \multicolumn{2}{|c|}{ Total } \\
\hline & (f) & $\%$ & (f) & $\%$ & (f) & $\%$ \\
\hline Doenças endócrinas, nutricionais e metabólicas (E00 - E90) & 18 & 10,8 & 88 & 53 & 106 & 63,8 \\
\hline Doenças do aparelho respiratório (J00 - J99) & 1 & 0,6 & 31 & 18,7 & 32 & 19,3 \\
\hline Algumas doenças infecciosas e parasitárias (A00 - B99) & 1 & 0,6 & 11 & 6,6 & 12 & 7,2 \\
\hline Doenças do aparelho digestivo (K00 - K93) & 2 & 1,2 & 4 & 2,4 & 6 & 3,6 \\
\hline Doenças do sangue e dos órgãos hematopoiéticos e alguns transtornos imunitários (D50 - D89) & 1 & 0,6 & 4 & 2,4 & 5 & 3 \\
\hline Algumas afecções originadas no período perinatal (P00 - P96) & 2 & 1,2 & 3 & 1,8 & 5 & 3 \\
\hline Total & 25 & 15,1 & 141 & 84,9 & 166 & 100 \\
\hline
\end{tabular}

$\mathrm{n}=$ número; $(\mathrm{f})=$ quantidade; $\%=$ porcentagem

Tabela 3: Classificação dos pacientes pediátricos, indígenas e não indígenas, conforme o diagnóstico principal de desnutrição ( $\mathrm{n}=100$ ). Mato Grosso, Brasil, 2012-2014.

\begin{tabular}{|c|c|c|c|c|c|c|}
\hline \multirow{2}{*}{ Diagnóstico de desnutrição segundo a CID - 10} & \multicolumn{2}{|c|}{ Não indígena } & \multicolumn{2}{|c|}{ Indígena } & \multicolumn{2}{|c|}{ Total } \\
\hline & (f) & $\%$ & (f) & $\%$ & (f) & $\%$ \\
\hline E43 Desnutrição proteico-calórica grave não especificada & 5 & 5 & 24 & 24 & 29 & 29 \\
\hline E44.0 Desnutrição proteico-calórica moderada & 4 & 4 & 19 & 19 & 23 & 23 \\
\hline E44.1 Desnutrição proteico-calórica leve & 5 & 5 & 9 & 9 & 14 & 14 \\
\hline E46 Desnutrição proteico-calórica não especificada & 4 & 4 & 30 & 30 & 34 & 34 \\
\hline Total & 18 & 18 & 82 & 82 & 100 & 100 \\
\hline
\end{tabular}

$\mathrm{n}=$ número; $(\mathrm{f})=$ quantidade; \%=porcentagem 
de cada grupo, visto que o número de indígenas internados por desnutrição no período analisado foi muito superior. Diferença estatística significante foi encontrada apenas entre os casos de infecção do sistema respiratório, visto que crianças indígenas têm quase cinco vezes mais chances de apresentarem infecção respiratória do que o não indígena $(\mathrm{p}<0,05)$.

As infecções respiratórias agudas (IRA) são as principais causas de internações e óbitos entre crianças indígenas menores de cinco anos ${ }^{15,16}$. Em um estudo com crianças Ianomâmis com pneumonia, foi visto que as chances de internação foram três vezes maiores entre aquelas com diagnóstico secundário de desnutrição, afirmando a relação desnutrição-IRA ${ }^{20}$. Esses dados atestam a elevada prevalência de infecções respiratórias entre indígenas observadas neste estudo, e confirmam que as infecções respiratórias permanecem entre as principais causas de hospitalização entre as crianças menores de cinco anos nessa população.

Quanto à antibioticoterapia empregada durante o tratamento, como pode ser visto na Tabela 6 , as crianças internadas por desnutrição têm quase três vezes mais chances de receberem até três antibióticos $(\mathrm{p}<0,05)$ do que um número superior a isso. $\mathrm{O}$ total de antibióticos prescritos por paciente variou de zero a dez; cinco pacientes não tiveram nenhum antimicrobiano prescrito e dois receberam dez antibióticos diferentes. A maior proporção dos pacientes, indígenas e não indígenas, receberam uma associação dos medicamentos. O maior número (43) recebeu dois medicamentos, seguidos por aqueles que receberam quatro (28) e três (27) antibióticos. Todos os pacientes que receberam um número de antibióticos superior a sete eram indígenas.
O Ministério da Saúde recomenda uma associação de até quatro antibióticos, dependendo da evolução clínica do quadro, para crianças desnutridas com letargia ou complicações como hipoglicemia, hipotermia ou sinais clínicos de infecção ${ }^{17}$. Embora haja estudos que apontem maior sobrevida de crianças desnutridas que recebem antibióticos durante o tratamento, a prática da prescrição de rotina tem sido questionada devido à ausência de ensaios clínicos que abordem o assunto, os custos onerosos do uso irracional de antimicrobianos, possíveis reações tóxicas, baixas proporções de infecções associadas a desnutrição leve e a alta resistência bacteriana ${ }^{4,21,22}$.

A politerapia antimicrobiana foi uma prática encontrada neste estudo, uma vez que $81,3 \%$ dos pacientes receberam associação de antibióticos, sendo recomendada em alguns casos, como relatado acima, entretanto, cabe aqui discutir a respeito do uso racional destes medicamentos diante das dificuldades supracitadas. Há poucas evidências que sustentem as recomendações atuais para o uso rotineiro de antibióticos ${ }^{3}$ em crianças desnutridas na ausência de complicações, e a segurança de uma abordagem sem o uso de antibióticos está relacionada a capacidade do profissional de saúde no diagnóstico preciso da desnutrição grave e suas complicações. No caso de locais com um sistema de saúde debilitado, o uso de antibióticos pode funcionar como uma rede de segurança, entretanto, a utilização desses medicamentos deve ser feita racionalmente, considerando seu impacto na saúde individual, coletiva e ao meio ambiente ${ }^{4,23}$

A resistência antimicrobiana (RAM) tornou-se um problema de saúde pública, que não deve ser ignorada por profissionais e

Tabela 4: Desfecho clínico dos pacientes pediátricos, indígenas e não indígenas, relacionado ao diagnóstico principal ( $n=166)$. Mato Grosso, Brasil, 2012-2014.

\begin{tabular}{|c|c|c|c|c|c|c|c|}
\hline \multirow{2}{*}{ Desfecho } & \multicolumn{4}{|c|}{ Diagnóstico } & \multirow[b]{2}{*}{ Outro } & \multirow{2}{*}{ Total } & \multirow{2}{*}{ Estatística } \\
\hline & E 46 & E 43 & E 44.1 & E 44.0 & & & \\
\hline Alta & 32 & 21 & 14 & 23 & 65 & 155 & \multirow{3}{*}{$\begin{array}{c}\chi^{2}=29,85 \\
p<0,01\end{array}$} \\
\hline Transferência & 0 & 4 & 0 & 0 & 0 & 4 & \\
\hline Óbito & 2 & 4 & 0 & 0 & 1 & 7 & \\
\hline Total & 34 & 29 & 14 & 23 & 66 & 166 & \\
\hline
\end{tabular}

$\mathrm{n}=$ número; $\chi^{2}=$ Chi-quadrado de Pearson.

Tabela 5: Frequência das infecções associadas à desnutrição encontradas em crianças indígenas e não indígenas ( $n=147)$. Mato Grosso, Brasil, 2012 - 2014.

\begin{tabular}{|c|c|c|c|c|c|c|c|}
\hline \multirow{2}{*}{ Infecções } & \multicolumn{2}{|c|}{ Não indígena } & \multicolumn{2}{|c|}{ Indígena } & \multicolumn{2}{|c|}{ Total } & \multirow{2}{*}{ Estatística* } \\
\hline & (f) & $(\%)$ & (f) & $(\%)$ & (f) & (\%) & \\
\hline Sistema digestório & 15 & 9,0 & 77 & 46,4 & 92 & 55,4 & 0,391 \\
\hline Sistema respiratório & 4 & 2,4 & 67 & 40,4 & 71 & 42,8 & $\begin{array}{c}0,002 \\
\text { OR: } 4,75\end{array}$ \\
\hline Sistema tegumentar & 2 & 1,2 & 22 & 13,3 & 24 & 14,5 & 0,256 \\
\hline Sistema cardiovascular & 0 & 0,0 & 2 & 1,2 & 2 & 1,2 & 0,721 \\
\hline Sistema urinário & 1 & 0,6 & 0 & 0 & 1 & 0,6 & 0,151 \\
\hline
\end{tabular}

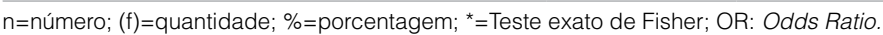


gestores dos sistemas de saúde por se tratar de onerosa e contribuir para inúmeros óbitos a cada ano ${ }^{24}$. Há dados disponíveis na literatura que apontam elevadas resistências aos antibióticos em populações de crianças desnutridas, e perante os dados encontrados nesse estudo, havendo pacientes que receberam até dez antibióticos diferentes, é preciso chamar a atenção para medidas políticas que podem contribuir para a contenção da RAM, como: reforço da vigilância e capacidade laboratorial, acesso ininterrupto a medicamentos essenciais garantido, aprimoramento da prevenção e do controle de infecções e promoção de inovação, pesquisa e desenvolvimento de novas ferramentas ${ }^{4,24}$.

A respeito da saúde indígena, considerando as insatisfatórias condições de saúde desta população e elevada prevalência de doenças infecciosas, o uso de antibióticos poderia ser considerado um sistema de segurança, no entanto, os pacientes indígenas foram os que receberam um maior número de antimicrobianos diferentes (até dez), chama-se a atenção para o uso empírico e possível negligência de culturas e antibiogramas. Nesses casos, medidas relacionadas a capacidade laboratorial e garantia de acesso contínuo aos medicamentos seriam de grande valia para a qualidade do tratamento e controle da $\mathrm{RAM}^{24}$.

Os dados apresentados a respeito do quantitativo de antibióticos prescritos podem estar relacionados a ausência de uma política de controle dos medicamentos prescritos e protocolos de uso de antimicrobianos no hospital onde foi realizado o estudo ${ }^{25}$. É preciso ressaltar que uma vigilância eficaz é fundamental para o controle da RAM e de possíveis efeitos adversos associados ao uso de antimicrobianos, havendo necessidade urgente de revisão das práticas prescritas referente à relação custo-benefício em cada caso, e de educação continuada aos profissionais envolvidos na prescrição, dispensação e administração dos medicamentos ${ }^{23,24}$.

Os resultados encontrados também revelam a necessidade de capacitação pelos profissionais cuja incumbência é o diagnóstico e a escolha da antibioticoterapia a ser empregada; bem como de todos os membros da equipe multiprofissional responsável pelos cuidados da criança desnutrida, a fim de diminuir o número de diagnósticos inespecíficos e evitar o uso indiscriminado de medicamentos antimicrobianos, prestando-se o cuidado individualizado e necessário a cada paciente.

Além da capacitação profissional, é imprescindível que haja recursos materiais e financeiros que possam atender as necessidades de crianças desnutridas hospitalizadas, como por exemplo, para garantir o acesso aos medicamentos adequados durante todo o período de internação, e para que sejam realizados exames como o antibiograma, indispensável para a escolha de antibióticos apropriados.

A elevada proporção de crianças indígenas desnutridas identificadas neste estudo soma-se várias outras pesquisas que evidenciam a insegurança alimentar e as desfavoráveis condições de saúde e nutrição entre a população indígena brasileira.

É necessário que a população infantil, indígena e não indígena, continue a ser acompanhada por meio de inquéritos nacionais voltados à saúde e nutrição, a fim de subsidiar a revisão e a elaboração de políticas públicas que atendam às suas necessidades e especificidades. Ações voltadas ao incentivo do aleitamento materno, para prevenção de doenças infecciosas agudas e desnutrição, dentre outros benefícios, também são necessárias e de baixo custo, podendo ser realizadas por meio de educação em saúde na atenção primária e com impacto positivo para a redução da morbimortalidade infantil.

\section{REFERÊNCIAS}

1. United Nations. The Millennium Development Goals Report 2015. New York: United Nations, 2015; p.14-23.

2. Horta BL, Santos RV, Welch JR, Cardoso AM, Santos JV, Assis AM, et al. Nutritional status of indigenous children: findings from the First National Survey of Indigenous Peoples Health and Nutrition in Brazil. Int J Equity Health. 2013;12:23. http://dx.doi.org/10.1186/1475-9276-12-23

3. World Health Organization (WHO). Guideline: updates on the management of severe acute malnutrition in infants and children. Geneva: World Health Organization, 2013.

4. Alcoba G, Kerac M, Breysse S, Salpeteur C, Galetto-Lacour A, et al. Do children with uncomplicated severe acute malnutrition need antibiotics? A systematic review and meta-analysis. PLoS One. 2013;8(1):e53184 http://dx.doi.org/10.1371/journal.pone.0053184

5. Trehan I, Goldbach HS, LaGrone LN, Meuli GJ, Wang RJ, Maleta $\mathrm{KM}$, et al. Antibiotics as part of the management of severe acute malnutrition. N Engl J Med. 2013;368(5):425-35. http://dx.doi.org/10.1056/NEJMoa1202851

6. Souza LG, Santos RV, Coimbra Jr CEA. Estrutura etária, natalidade e mortalidade do povo indígena Xavante de Mato Grosso,
Amazônia, Brasil. Cienc Saude Coletiva. 2010;15(Supl. 1): 1465-73.

http://dx.doi.org/10.1590/S1413-81232010000700058

7. Barreto CTG, Cardoso AM, Coimbra Jr CEA. Estado nutricional de crianças indígenas Guarani nos estados do Rio de Janeiro e São Paulo, Brasil. Cad Saude Publica. 2014;30(3):657-62. http://dx.doi.org/10.1590/0102-311X00117813

8. Coimbra Jr. CEA. Saúde e povos indígenas no Brasil: reflexões a partir do I Inquérito Nacional de Saúde e Nutrição Indígena. Cad Saude Publica. 2014;30(4):855-9. http://dx.doi.org/10.1590/0102-311X00031214

9. Instituto Brasileiro de Geografia e Estatística. Características gerais dos indígenas: resultados do universo. Rio de Janeiro: IBGE, 2010; p.244

10. Reichardt FV, Garavello MEPE. Quando habitar corresponde ao direito humano à alimentação. Revista de direito internacional 2017;14(1):68-79.

11. Castro GTM, Kaufer-Horwitz M, Carrillo-López HA, Klünder-Klünder M, Jarillo-Quijada A, García-Hernández HR. Estado nutricional de niños en condiciones críticas de ingreso a las unidades de terapia intensiva pediátrica. Bol Med Hosp Infant Mex. 2013;70(3):216-21. 
12. Dias MCAP, Freire LMS, Franceschini LCC. Recomendações para alimentação complementar de crianças menores de dois anos. Rev Nutr. 2010;23(3):475-86. http://dx.doi.org/10.1590/S1415-52732010000300015

13. Machado AKF, Elert VW, Pretto ADB, Pastore CA. Intenção de amamentar e de introdução de alimentação complementar de puérperas de um Hospital-Escola do sul do Brasil. Cienc Saúde Coletiva. 2014;19(7):1983-9

http://dx.doi.org/10.1590/1413-81232014197.03162013

14. Pedraza DF, Araujo EMN. Internações das crianças brasileiras menores de cinco anos: revisão sistemática da literatura. Epidemiol Serv Saúde. 2017;26(1):169-82. http://dx.doi.org/10.5123/s1679-49742017000100018

15. Patzer JD, Menegolla IA. Hospitalização de crianças indígenas de etnia Guarani, Distrito Sanitário Especial Indígena Litoral Sul, Rio Grande do Sul. Tempus Actas Saúde Coletiva. 2013;7(4):195-204. http://dx.doi.org/10.18569/tempus.v7i4.1429

16. Lunardi R, Santos RV, Coimbra Jr CEA. Morbidade hospitalar de indígenas Xavante, Mato Grosso, Brasil (2000-2002). Rev Bras Epidemiol. 2007;10(4):441-52. http://dx.doi.org/10.1590/S1415-790X2007000400002

17. Brasil. Ministério da Saúde. Secretaria de Atenção à Saúde. Coordenação Geral da Política de Alimentação e Nutrição. Manual de atendimento da criança com desnutrição grave em nível hospitalar. Brasília: Ministério da Saúde; 2005.

18. Cantón SBF, Uribe RV. La mortalidad por desnutrición en México en menores de cinco años, 1990-2009. Bol Med Hosp Infant Mex. 2010;67(5):471-3.
19. Lima AM, Gamallo SMM, Oliveira LFC. Desnutrição energéticoproteica grave durante a hospitalização: aspectos fisiopatológicos e terapêuticos. Rev Paul Pediatr. 2010;28(3):353-61. http://dx.doi.org/10.1590/S0103-05822010000300015

20. Caldart RV, Marrero L, Basta PC, Orellana JDY. Fatores associados à pneumonia em crianças Yanomami internadas por condições sensíveis à atenção primária na região norte do Brasil. Cien Saude Coletiva. 2016;21(5):1597-1606. http://dx.doi.org/10.1590/1413-81232015215.08792015

21. Lazzerini M, Tickell D. Antibiotics in severely malnourished children: systematic review of efficacy, safety and pharmacokinetics. Bull World Health Organ. 2011;89(8):593-606. http://dx.doi.org/10.2471/BLT.10.084715

22. Manary MJ, Maleta K, Trehan I. Randomized, double-blind, placebo-controlled trial evaluating the need for routine antibiotics as part of the outpatient management of severe acute malnutrition. Washington: Bridge, 2012; p.6-11.

23. Oliveira KR, Munaretto P. Uso racional de antibióticos: responsabilidade de prescritores, usuários e dispensadores. Rev Contexto Saúde. 2013;10(18):43-51. https://doi.org/10.21527/2176-7114.2010.18.43-51

24. Organização Mundial de Saúde (OMS). A crescente ameaça da resistência antimicrobiana opções de ação. Genebra: Organização Mundial de Saúde, 2012; p. 6-12.

25. Silva ERM. Análise do perfil das prescrições de antimicrobianos na clínica médica de um hospital público do Pará. Rev Bras Farm Hosp Serv Saúde. 2012;3(2):15-19. 